\title{
A Cell-Electrode Interface Noise Model for High-density Microelectrode Arrays
}

\author{
Neil Joye, Student Member, IEEE, Alexandre Schmid, Member, IEEE, and Yusuf Leblebici, Senior \\ Member, IEEE
}

\begin{abstract}
A cell-electrode interface noise model is developed which is dedicated to enable the co-simulation of the cellelectrode electrical characteristics, along with the electronics of novel CMOS-based MEA. The electrode noise is investigated for Pt and Pt black electrodes. It is shown that the electrode noise can be the dominant noise source in the full system. Moreover, Pt black electrodes benefit from up to $5 \mu \mathbf{V}_{\text {rms }}$ decrease of the electrode output noise, for small electrodes. Furthermore, the cell-electrode interface noise spectral density is shown to be $10 \mathrm{~dB}$ to $20 \mathrm{~dB}$ larger at $1 \mathrm{kHz}$ when a cell is lying on top of the electrode. This increase depends on the neural cell adhesion on the MEA surface.
\end{abstract}

\section{INTRODUCTION}

$\mathrm{M}$ ICROELECTRODE arrays (MEAs) have become essential for monitoring electrogenic cells. They provide a significant tool for measuring neural activity of cells cultured in-vitro [1]. However, one of the main limitations of MEAs is related to their low spatial resolution. Commercial MEAs usually contain approximately 60 electrodes, in total, with electrode sizes ranging from $10 \mu \mathrm{m}$ to $50 \mu \mathrm{m}$, and inter-electrode spacings of up to $100 \mu \mathrm{m}$. These dimensions are much larger than the 10-20 $\mu \mathrm{m}$ typical size of vertebrate neurons used during electrophysiological experiments. However recently, a new generation of CMOSbased MEAs containing a high-density of sensors has emerged [2]-[4]. These new devices take the advantage of CMOS electronics, which offers on-chip multiplexing, amplification, and filtering, for handling a large number of closely spaced electrodes. Thus, CMOS-based MEAs have a pitch dimension as low as $7.8 \mu \mathrm{m}$ with an electrode diameter of $4.5 \mu \mathrm{m}$ [2]. It is therefore now possible to carry out experiments at subcellular resolution.

The design of these novel CMOS-based MEAs demands accurate compact electrical models of the cell-electrode interface in a format enabling co-simulation with the CMOS circuits. Thus the electrical characteristics, such as noise or power consumption, of CMOS-based MEAs could be modeled and simulated as a whole system including the cellelectrode interface and the electronics.

In this scope, an electrical model of the cell-electrode

This work has been conducted with the support of the Swiss NSF grant number 205321-116780.

N. Joye, A. Schmid, and Y. Leblebici are with the Microelectronic Systems Laboratroy, Swiss Federal Institute of Technology (EPFL), 1015 Lausanne, Switzerland (phone: +41 -21 6936927; fax: +41 -21 6936959; email: neil.joye@epfl.ch). interface for recording neural activity from high-density MEAs has been developed by the authors [5]. However, the noise analysis of the cell-electrode interface has not been taken into account in this earlier analysis. Thus, the scope of this work consists of developing a cell-electrode interface noise model for high-density MEAs. The noise model is described in Section II, and simulation results are presented in Section III.

\section{THE MOdels}

The point-contact model depicted in Fig. 1 is considered in this study [5]. It is the standard model used to describe the electrical characteristics of the cell-electrode interface.

The noise model developed in this Section takes into account the noise from the electrode and the electrolyte solution. Noise arising from the neuron cell such as channel noise is not considered in this study [6], which can not appropriately be described by the noise of electrical circuit elements such as resistors and capacitors. Thus, the equivalent circuit of the attached membrane $\left(R_{m}, C_{m}\right.$, and $\mathrm{C}_{\mathrm{hd}}$ ) is not described in this Section [5].

\section{A. The Point-Contact Model}

The sealing resistance $R_{\text {seal }}$, as depicted in Fig. 1, represents the resistance between the cleft and the surrounding solution. It is described as

$R_{\text {seal }}=\frac{\rho_{s}}{\theta \pi d}$

where $\rho_{s}$ is the resistivity of the electrolyte ( $1 \Omega \mathrm{m}$ for typical solutions), $d$ is the cell-electrode distance, and $\theta$ is a correction factor related to geometry which is fixed to 5.78 [5].

The equivalent circuit of the electrode is based on an electrode model, which is typically used to describe the electrode-electrolyte impedance as described in [7]. It includes a constant phase angle impedance $Z_{C P A}$, which represents the interface capacitance, in parallel with a charge transfer resistance $R_{c t}$, both in series with a spreading resistance $R_{\text {spread }}$. The Warburg impedance due to diffusion of the chemical reactants in solution is not included in this model. It is negligible for the materials and frequency range used in electrophysiological experiments.

The constant phase angle impedance $Z_{C P A}$ is described as

$Z_{C P A}=\frac{1}{\left(j \omega C_{d l}\right)^{n}}$ 


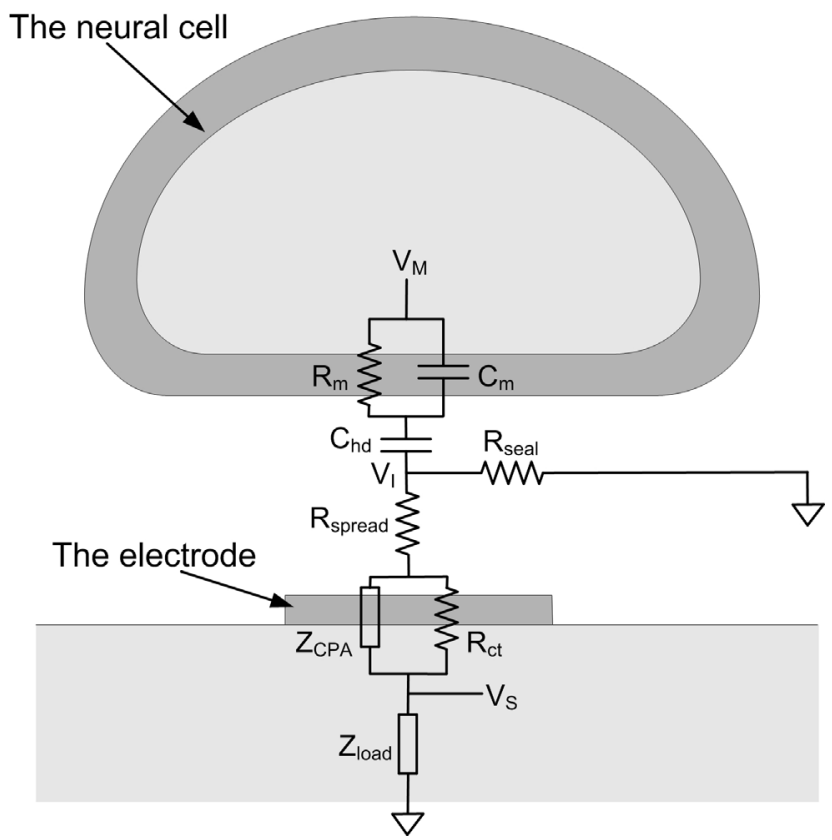

Fig. 1. Point-contact model of the cell-electrode interface (not to scale).

where $C_{d l}$ is the double layer capacitance, and $n$ is an empirical factor which value is between 0 and 1 , and related to surface irregularities [5].

The charge-transfer resistance $R_{c t}$ represents the faradic process where charges transfer between the electrode and the electrolyte by means of oxidation-reduction reactions. For small values of overpotential during a faradic charge transfer, $R_{c t}$ may be expressed as

$R_{c t}=\frac{k T}{q} \frac{1}{z J_{0} A_{e l}}$

where $z$ is the number of electrons involved in the oxidationreduction reaction, $J_{0}$ is the equilibrium exchange current density, and $A_{e l}$ is the electrode surface.

The spreading resistance $R_{\text {spread }}$ represents the resistance to the current spreading from the electrode to the counter electrode. Assuming that the counter electrode is infinitely large, the spreading resistance is given in [7] by

$$
R_{\text {spread }}=\frac{\rho_{s}}{4 R_{e l}}
$$

for circular electrodes ( $R_{e l}$ is the radius of the electrode).

The load impedance $Z_{\text {load }}$ represents the load impedance of the cell-electrode interface system. In CMOS-based MEAs, it is usually equal to the input impedance of the amplification stage.

\section{B. The Cell-Electrode Interface Noise Model}

The noise model developed in this Section is depicted in Fig. 2 and covers two cases: case one, when no neural cell lies on top of the electrode as depicted in Fig. 2(a), and case two, when one cell covers the entire surface of the electrode as described in Fig. 2(b).

The electrode is described by $Z_{e l}$, which is the electrode impedance, and $V_{\text {el,noise }}$, which is the electrode noise voltage.

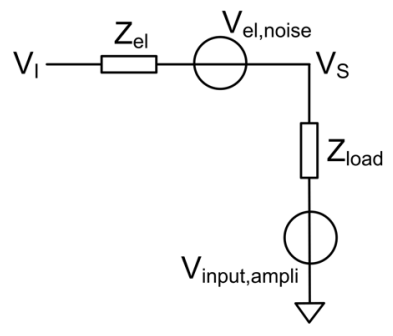

(a) Without cell

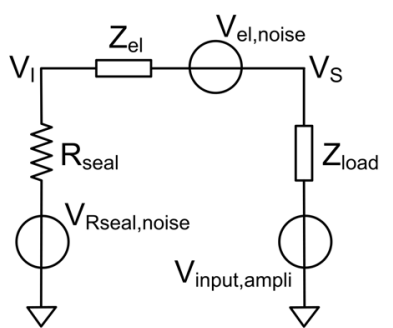

(b) With cell
Fig. 2. Equivalent cell-electrode interface noise model for (a) no cell and (b) one cell lying on top of the electrode.

For Pt electrodes, thermal noise has been empirically shown to be the dominant noise source [8], [9]. Thus, the electrode is considered to be nonfaradaic and the nonequilibrium excess noise caused by charge transfer processes produced by electrochemical interactions at the electrode surface is not taken into account [10].

Thus, $Z_{e l}$ and $V_{e l, n o i s e}$ are expressed as

$$
\begin{aligned}
& Z_{e l}(j \omega)=\frac{\left(R_{c t}+R_{\text {spread }}\right)+R_{c t} R_{\text {spread }}\left(j \omega C_{d l}\right)^{n}}{1+R_{c t}\left(j \omega C_{d l}\right)^{n}} \\
& V_{\text {el,noise }}=\sqrt{4 k T R_{N} \Delta f}\left[V_{r m s}\right]
\end{aligned}
$$

where $R_{N}$ is the equivalent noise resistance that is equal to the real part of the electrode impedance magnitude, and $\Delta f$ is the recording bandwidth [8], [11].

The seal resistance, which appears when a cell lies on top of the electrode, is described by $R_{\text {seal }}$ and $V_{\text {Rseal,noise, which is }}$ the seal resistance noise voltage, expressed in the following equation

$V_{\text {Rseal, noise }}=\sqrt{4 k T R_{\text {seal }} \Delta f} \quad\left[V_{r m s}\right]$

The input referred noise of the amplification stage of a CMOS-based MEA is represented by $V_{\text {input,ampli }}$.

In order to be able to compare the electrode and electrolyte solution noise with the noise of the CMOS circuitry, the equivalent noise of the cell-electrode interface at node $V_{S}$ needs to be calculated. Thus, for the case where no cell lies on top of the electrode, the electrode noise spectral density at node $V_{S}$ is expressed as in (8). For the second case, where one cell entirely covers the surface of the electrode, the electrode and electrolyte solution noise spectral density at node $V_{S}$ is described as in (9).

$$
\begin{aligned}
& V_{S, n o i s e}=\frac{Z_{\text {load }}}{Z_{\text {el }}+Z_{\text {load }}} \sqrt{4 k T R_{N}}\left[\frac{V}{\sqrt{H z}}\right] \\
& V_{S, \text { noise }}=\frac{Z_{\text {load }}}{Z_{\text {el }}+R_{\text {seal }}+Z_{\text {load }}} \sqrt{4 k T\left(R_{N}+R_{\text {seal }}\right)}\left[\frac{V}{\sqrt{H z}}\right]
\end{aligned}
$$

The cell-electrode interface noise is maximum when $Z_{\text {load }}$ is much larger than $Z_{e l}$ and $R_{\text {seal }}$. Moreover, amplification stages with very large input impedances are usually designed for CMOS-based MEAs [12]. Thus the worst case, where $Z_{\text {load }}$ is infinite, is considered in this study. 


\section{Simulation Results}

The cell-electrode interface noise characteristics described in Section II are simulated under Matlab and using the numerical values detailed in the following. Considering a $\mathrm{Pt}$ electrode, the values given in [7] are considered for $Z_{C P A}$, $C_{d l}$, and $R_{c t} ; d_{O H P}$ is equal to $0.5 \mathrm{~nm}, d_{I H P}$ is neglected, $\varepsilon_{O H P}=$ $78, z=4, n^{0}=154 \mathrm{nM}$, and $n=0.9$ [5].

The electrode noise spectral density of a Pt electrode, as described in Section II, and considering different electrode radius is depicted in Fig. 3(a). The electrode noise spectral density has an approximate dependence of $-10 \mathrm{~dB} / \mathrm{dec}$ for small frequencies. However, for frequencies higher than 1$10 \mathrm{kHz}, R_{N}$ is dominated by the spreading resistance $R_{\text {spread }}$. Thus, the electrode noise spectral density is constant for high frequencies. Moreover, when the electrode size is decreased, the electrode noise spectral density increases due to an increase of the electrode impedance as shown in (2)(5).

In order to reduce the electrode noise, the electrode impedance needs to be decreased. One solution is to increase the electrode size. However, this leads to an increase of the pitch dimension in the electrode array. Thus, a solution which is also acceptable for high-density MEAs consists of using electrode materials such as Pt black or TiN. These electrodes have a much higher electrode-electrolyte contact surface while having the same geometric area as $\mathrm{Pt}$ electrodes. This leads to an approximate two order of magnitude decrease of the electrode impedance [7]. The comparison of the electrode noise spectral density for Pt and Pt black electrodes is depicted in Fig. 3(b). The electrode radius is fixed to $1 \mu \mathrm{m}$ and $25 \mu \mathrm{m}$. In order to simulate $\mathrm{Pt}$ black electrodes, the electrode surface $A_{e l}$ is increased by two orders of magnitude. The other electrode parameters are the same as for Pt electrodes. Thus, the goal of this approach is not to simulate the exact Pt black electrode characteristics, but to have an approximation of its electrical behavior compared to Pt electrodes. As shown in Fig. 3(b), Pt black electrodes have an electrode noise spectral density reduction up to $20 \mathrm{~dB}$ for small frequencies. However, no noise reduction is achieved for frequencies higher than 10-100 $\mathrm{kHz}$. This is due to the fact that $R_{\text {spread }}$ depends on the electrode geometric area as described in (4). Similar results are found with TiN electrodes [7].

In order to compare the electrode noise with the input referred noise of typical amplification stages given in literature [11]-[13], the electrode output noise, where its spectral density is expressed in (8), is depicted in Fig. 4 versus the electrode size. However, most input referred noise values found in literature are measured over different bandwidths. Therefore, the electrode output referred noise is calculated in this work considering two different bandwidths: $1 \mathrm{~Hz}$ to $100 \mathrm{kHz}$ [3] and $100 \mathrm{~Hz}$ to $10 \mathrm{kHz}$ [11]. As shown in Fig. 4, the electrode output noise increases when it is calculated over a larger bandwidth. Moreover, as explained earlier, electrode noise is decreased
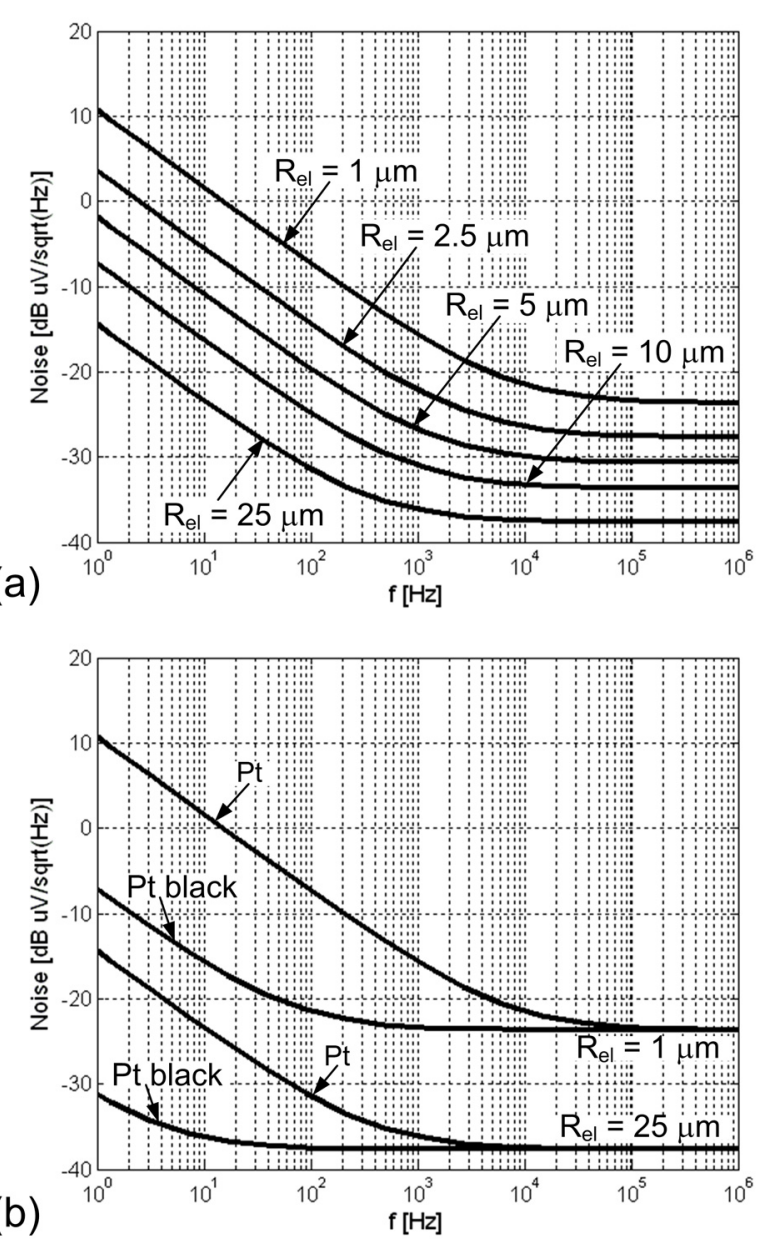

Fig. 3. Electrode noise spectral density for (a) different electrode radius and (b) different electrode materials (Pt and Pt black). A Pt electrode is considered in (a) and an electrode radius of $1 \mu \mathrm{m}$ and $25 \mu \mathrm{m}$ is used in (b).

with larger electrodes.

The electrode output noise is also depicted in Fig. 4 for Pt and Pt black electrodes. For electrodes which have a diameter larger than $30 \mu \mathrm{m}$, the noise reduction offered by Pt black electrodes is negligible. However for small electrodes, Pt black electrodes enable up to $5 \mu \mathrm{V}_{\text {rms }}$ decrease of the electrode output noise when the electrode radius is 1 $\mu \mathrm{m}$. Thus, Pt black electrodes are especially useful for decreasing the electrode noise in high-density MEAs.

Most amplification stages found in the literature have input referred noise $V_{\text {input,ampli }}$ smaller than $10 \mu \mathrm{V}_{\text {rms }}$ [3], [11]-[13]. As shown in Fig. 4, the electrode output noise can be larger than this value when the electrode has a diameter smaller than $10 \mu \mathrm{m}$. Thus, careful consideration has to be devoted to the noise generated by the electrode-electrolyte interface when designing high-density CMOS-based MEAs.

The electrode noise spectral density at node $V_{S}$, as expressed in (8) and (9), with a cell lying on top of an electrode is depicted in Fig. 5. A typical neural cell diameter of $10 \mu \mathrm{m}$ covering the whole surface of an electrode with a radius of $5 \mu \mathrm{m}$ is considered. As shown in Fig. 5, the 


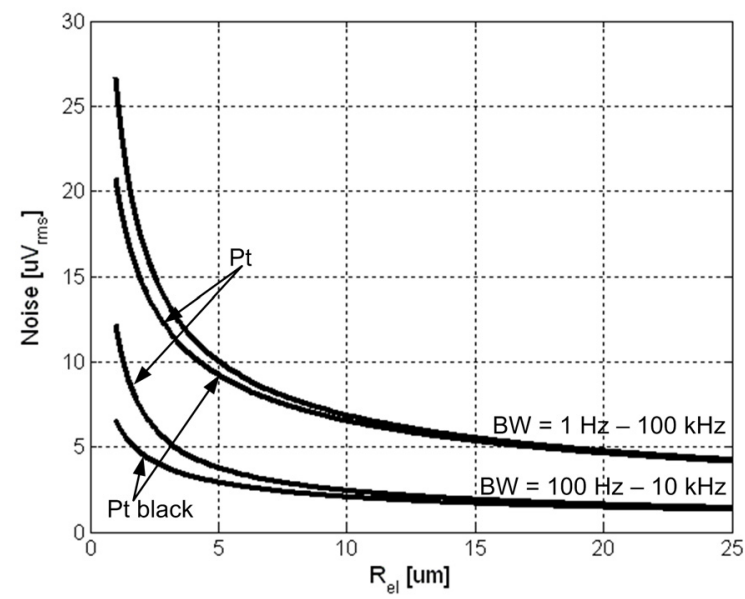

Fig. 4. Electrode output noise versus the electrode radius for Pt and Pt black electrodes. The output referred noise is calculated over two different bandwidths $(1 \mathrm{~Hz}-100 \mathrm{kHz}$ and $100 \mathrm{~Hz}-10 \mathrm{kHz})$.

electrode noise spectral density is increased compared to the case where no cell lies of top of the electrode surface. Similar results have been demonstrated using electrolyteoxide-silicon field effect transistors (EOSFET) [14]. This increase depends on the cell-electrode average distance $d$ and on the frequency. For strong cell adhesion on the electrode surface, which is modeled as a cell-electrode distance of $10 \mathrm{~nm}$, the electrode noise spectral density increases due to a larger seal resistance $R_{\text {seal }}$ as expressed in (1). In this case, the electrode noise spectral density at $1 \mathrm{kHz}$ is $15-20 \mathrm{~dB}$ larger than the noise spectral density without a cell, as depicted in Fig. 5. This property can be used to measure neural cell adhesion on the surface of the electrode [14].

\section{CONCLUSION}

A cell-electrode interface noise model is developed in order to enable the co-simulation of the cell-electrode electrical characteristics along with the electronic circuits of novel CMOS-based MEAs. For high-density MEAs and small $\mathrm{Pt}$ electrodes, it has been shown that the electrode noise can be the largest noise source of the whole system. A solution consists of using $\mathrm{Pt}$ black or TiN electrode materials. The electrode noise is thus decreased whereas having the same electrode geometric area. The choice of the frequency bandwidth has also been demonstrated to play a significant role when calculating and measuring the electrode output noise. Furthermore, the cell-electrode interface noise spectral density is shown to be $10 \mathrm{~dB}$ to 20 $\mathrm{dB}$ larger, at $1 \mathrm{kHz}$ and higher, when a cell lies on top of the electrode. This value depends on the cell adhesion and could be used to measure the neuron adhesion on the surface of the MEA [14].

Finally, electrical measurements need to be performed on fabricated MEAs in order to confirm the simulation results obtained with the noise model presented in this paper.

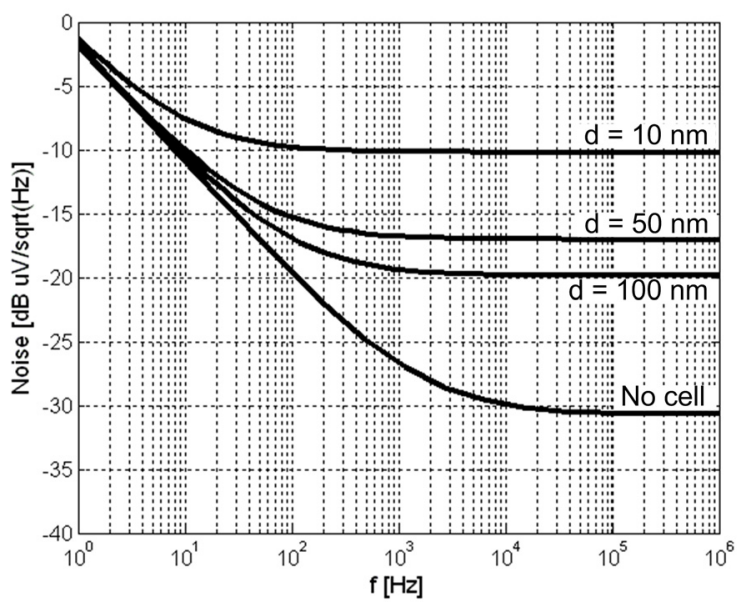

Fig. 5. Electrode noise spectral density with and without a cell covering the electrode. For the case where a cell is lying on the surface of the electrode, three different cell-electrode distances are used $(10 \mathrm{~nm}, 50 \mathrm{~nm}$, and $100 \mathrm{~nm}$ ). A cell diameter of $10 \mu \mathrm{m}$ and an electrode radius of $5 \mu \mathrm{m}$ are considered.

\section{REFERENCES}

[1] S. Marom and G. Shahaf, "Development, learning and memory in large random networks of cortical neurons: lessons beyond anatomy," Quarterly Reviews of Biophysics, vol. 35, no. 1, pp. 63-87, May 2002.

[2] B. Eversmann et al., "A $128 \times 128$ CMOS biosensor array for extracellular recording of neural activity," IEEE J. Solid-State Circuits, vol. 38, no. 12, pp. 2306-2317, Dec. 2003.

[3] U. Frey et al., "An 11k-electrode 126-channel high-density microelectrode array to interact with electrogenic cells," in Proc. IEEE ISSCC 2007, pp. 158-593.

[4] K. Imfeld et al., "Large-scale, high-resolution data acquisition system for extracellular recording of electrophysiological activity," IEEE Trans. Biomed. Eng., vol. 55, no. 8, pp. 2064-2073, Aug. 2008.

[5] N. Joye, A. Schmid, and Y. Leblebici, "An electrical model of the cellelectrode interface for high-density microelectrode arrays," in Proc. 30th Annu. Int. IEEE EMBS Conf., Vancouver, 2008, pp. 559-562.

[6] A. A. Faisal, L. P. J. Selen, and D. M. Wolpert, "Noise in the nervous system," Nat. Rev. Neurosci., vol. 9, no. 4, pp. 292-303, Apr. 2008.

[7] W. Franks, I. Schenker, P. Schmutz, and A. Hierlemann, "Impedance characterization and modeling of electrodes for biomedical applications," IEEE Trans. Biomed. Eng., vol. 52, no. 7, pp. 12951302, July 2005.

[8] R. C. Gesteland, B. Howland, J. Y. Lettvin, and W. H. Pitts, "Comments on microelectrodes," Proc. IRE, vol. 47, no. 11, pp. 18561862, Nov. 1959.

[9] X. Liu, A. Demosthenous, and N. Donaldson, "Platinum electrode noise in the ENG spectrum," Med. Biol. Eng. Comput., vol. 46, no. 10, pp. 997-1003, Oct. 2008.

[10] A. Hassibi, R. Navid, R. W. Dutton, and T. H. Lee, "Comprehensive study of noise processes in electrode electrolyte interfaces," J. Appl. Phys., vol. 96, no. 2, pp. 1074-1082, July 2004.

[11] P. Mohseni and K. Najafi, "A fully integrated neural recording amplifier with DC input stabilization," IEEE Trans. Biomed. Eng., vol. 51, no. 5, pp. 832-837, May 2004.

[12] B. Gosselin, M. Sawan, and C. A. Chapman, "A low-power integrated bioamplifier with active low-frequency suppression," IEEE Trans. Biomed. Circuits and Systems, vol. 1, no. 3, pp. 184-192, Sep. 2007.

[13] R. R. Harrison and C. Charles, "A low-power low-noise CMOS amplifier for neural recording applications," IEEE J. Solid-State Circuits, vol. 38, no. 6, pp. 958-965, June 2003.

[14] M. Voelker and P. Fromherz, "Nyquist noise of cell adhesion detected in a neuron-silicon transistor," Physical Review Letters, vol. 96, no. 22, June 2006. 